\title{
Measurements of ultracold neutron upscattering and absorption in polyethylene and vanadium
}

\author{
E. I. Sharapov, ${ }^{1}$ C. L. Morris,${ }^{2,}{ }^{*}$ M. Makela, ${ }^{2}$ A. Saunders, ${ }^{2}$ Evan R. Adamek, ${ }^{3}$ Y. Bagdasarova, ${ }^{2}$ L. J. Broussard, ${ }^{2}$ \\ C. B. Cude-Woods, ${ }^{3}$ Deion E. Fellers, ${ }^{2}$ Peter Geltenbort, ${ }^{4}$ S. I. Hasan,,${ }^{5}$ K. P. Hickerson, ${ }^{6}$ G. Hogan, ${ }^{2}$ A. T. Holley, ${ }^{3}$ \\ Chen-Yu Liu, ${ }^{3}$ M. P. Mendenhall, ${ }^{6}$ J. Ortiz, ${ }^{2}$ R. W. Pattie, Jr., ${ }^{7}$ D. G. Phillips II, ${ }^{7}$ J. Ramsey, ${ }^{2}$ D. J. Salvat, ${ }^{3}$ S. J. Seestrom, ${ }^{2}$ \\ E. Shaw, ${ }^{2}$ S. K. L. Sjue, ${ }^{2}$ W. E. Sondheim, ${ }^{2}$ B. VornDick, ${ }^{7}$ Z. Wang, ${ }^{2}$ T. L. Womack, ${ }^{2}$ A. R. Young, ${ }^{7}$ and B. A. Zeck $^{7}$ \\ ${ }^{1}$ Joint Institute for Nuclear Research, 141980 Dubna, Russia \\ ${ }^{2}$ Los Alamos National Laboratory, Los Alamos, New Mexico 87544, USA \\ ${ }^{3}$ Department of Physics, Indiana University, Indiana 47405-7105, USA \\ ${ }^{4}$ Institut Laue-Langevin, 38042 Grenoble Cedex 9, France \\ ${ }^{5}$ Department of Physics and Astronomy, University of Kentucky, Lexington, Kentucky 40506, USA \\ ${ }^{6}$ Kellogg Radiation Laboratory, California Institute of Technology, Pasadena, California 91125, USA \\ ${ }^{7}$ Department of Physics, North Carolina State University, Raleigh, North Carolina 27695, USA \\ (Received 5 June 2013; revised manuscript received 24 July 2013; published 3 September 2013)
}

\begin{abstract}
The study of neutron cross sections for elements used as efficient "absorbers" of ultracold neutrons (UCN) is crucial for many precision experiments in nuclear and particle physics, cosmology and gravity. In this context, "absorption" includes both the capture and upscattering of neutrons to the energies above the UCN energy region. The available data, especially for hydrogen, do not agree between themselves or with the theory. In this report we describe measurements performed at the Los Alamos National Laboratory UCN facility of the UCN upscattering cross sections for vanadium and for hydrogen in $\mathrm{CH}_{2}$ using simultaneous measurements of the radiative capture cross sections for these elements. We measured $\sigma_{\text {up }}=1972 \pm 130 \mathrm{~b}$ for hydrogen in $\mathrm{CH}_{2}$, which is below theoretical expectations, and $\sigma_{\text {up }}=25 \pm 9 \mathrm{~b}$ for vanadium, in agreement with the expectation for the neutron heating by thermal excitations in solids.
\end{abstract}

DOI: 10.1103/PhysRevC.88.037601

PACS number(s): 25.40.Fq, 25.40.Kv, 28.20.Ka

Several recent reviews highlight the application of ultracold neutrons in past and ongoing experiments for searching for the neutron electric dipole moment [1], for precision measurement of the neutron lifetime [2], for neutron $\beta$-decay correlations [3], and for gravitational quantum states [4], as well as for solving new issues in cosmology [5]. Ultracold neutrons greatly increase the sensitivity of experiments because they can be confined in the apparatus for times comparable with the neutron lifetime owing to the action of the 'optical' Fermi potential, $U_{F}$,

$$
U_{F}=\frac{2 \pi \hbar^{2}}{m} N b,
$$

where $m$ is the neutron mass, $N$ is the number density of the material, and $b$ is the neutron scattering length.

Materials with high positive potential, $U_{F} \simeq 250 \mathrm{neV}$, are used for trapping UCN because neutrons with kinetic energy lower than that value are reflected from walls at all angles of incidence. The velocity of such neutrons is below $7 \mathrm{~m} / \mathrm{s}$. On the other hand, materials made from elements with a small negative optical potential, like hydrogen, vanadium, titanium serve for removal of UCN through absorption and/or upscattering to higher energy. The efficiency of this removal is important for many measurements and this continues to

\footnotetext{
*Corresponding author: morris@lanl.gov

Published by the American Physical Society under the terms of the Creative Commons Attribution 3.0 License. Further distribution of this work must maintain attribution to the author(s) and the published article's title, journal citation, and DOI.
}

motivate the study of such materials. Vanadium and hydrogen are perfect neutron incoherent scatterers, and both have small negative, practically non-reflective potential $U_{F} \simeq-7.1 \mathrm{neV}$. For these elements, the theory of upscattering in one-phonon incoherent approximation is usually applied in the theory of upscattering, and the UCN isotropic differential upscattering cross section for cubic lattices can be calculated by a simple formula [6]:

$$
\frac{d \sigma_{\mathrm{up}}}{d E}=\sigma_{b} \sqrt{\frac{E}{E_{i}}}\left(e^{E / k T}-1\right)^{-1} \frac{g(E)}{A} e^{-2 W},
$$

where $\sigma_{b}=4 \pi b^{2}$ is the cross section for bound nuclei with the mass number $A, E_{i}$ is the initial UCN energy, $E$ is the energy after upscattering, $g(E)$ is the phonon density of states, and the last exponent is the Debye-Waller factor which is often measured, although it can be calculated as well. The MCNP code [7] with the thermal data kernels for the scattering law $S(\alpha, \beta)$ ( $\alpha$ and $\beta$ are the reduced momentum and energy transfers) calculates the neutron inelastic scattering cross section corrected for multiphonon contributions to Eq. (2) when applicable. The cross sections for several neutron moderators have been calculated using MCNP by [8]. For polyethylene the upscattering cross section is $\sigma_{\text {up }}=320 \mathrm{~b}$ at the minimum evaluated energy of $0.01 \mathrm{meV}(v=43.7 \mathrm{~m} / \mathrm{s})$. Applying the $1 / v$ law below $\simeq 0.01 \mathrm{meV}$ leads to the value of $3500 \mathrm{~b}$ at a velocity of $4 \mathrm{~m} / \mathrm{s}$.

Total neutron cross sections for vanadium have been measured over the velocity range from $4.4 \mathrm{~m} / \mathrm{s}$ to $17.7 \mathrm{~m} / \mathrm{s}$ in [9]. The inferred capture cross section was analyzed together with other thermal data and was shown to follow the expected 
$1 / v$ law up to thermal velocities, $2200 \mathrm{~m} / \mathrm{s}$. With this result and with the thermal value $\sigma_{a}=4.92 \pm 0.05 \mathrm{~b}$ [10] for the isotope ${ }^{51} \mathrm{~V}$, we obtain $\sigma_{a} \sqrt{E}=0.808 \pm 0.008 \mathrm{~b} \mathrm{eV}^{1 / 2}$. The vanadium upscattering cross section $\sigma_{\text {up }}$ has not been measured but has been estimated [11] in the one-phonon approximation to follow the $1 / v$ law below $\simeq 0.1 \mathrm{meV}$ with the value $\sigma_{\text {up }} \sqrt{E}=0.012 \pm 0.002 \mathrm{~b} \mathrm{eV}^{1 / 2}$, giving the relative contribution of the upscattering cross section at a level of $1.5 \%$. The capture cross section for ${ }^{51} \mathrm{~V}$ is large at UCN energies, $2700 \mathrm{~b}$ for UCN at $4 \mathrm{~m} / \mathrm{s}$ velocity. For hydrogen the relationship between absorption and upscattering is reversed: the average UCN upscattering cross section, according to the measurement [12], is $3745 \pm 370 \mathrm{~b}$ while the thermal cross section value $\sigma_{a}=0.3326 \pm 0.0007 \mathrm{~b}$ [10] and the $1 / v$ law leads to $\sigma_{a}(4 \mathrm{~m} / \mathrm{s})=182.9 \pm 0.4 \mathrm{~b}$. Lower upscattering cross sections values for hydrogen in polyethylene have been obtained from the recent UCN time-of-flight measurements [13], where the $\sigma_{\text {up }}(E)$ was shown to follow the $1 / v$ law with the value $\sigma_{\text {up }}(4 \mathrm{~m} / \mathrm{s})=2053 \pm 40 \mathrm{~b}$, at $4 \mathrm{~m} / \mathrm{s}$. The reasons for the disagreement between the results of measurements [12] and [13] remain unclear but might be resolved if the mean velocity of the measurement from [12] was specified.

Measurements of the UCN upscattering and capture cross sections have been performed using the Los Alamos National Laboratory solid-deuterium ultracold neutron source driven by an $800-\mathrm{MeV}, 5 \mu \mathrm{A}$ average proton beam provided by the Los Alamos Neutron Science Center (LANSCE) linear accelerator. The source is described in detail in a recent publication by Saunders et al. [14]. The UCN density in the stainless steel guide after the source exit window was $\simeq 1 \mathrm{UCN} / \mathrm{cm}^{3}$. The UCN velocity spectrum was cut off by the $U_{F}=189 \mathrm{neV}$ potential. Our Monte Carlo modeling of the UCN transport in the source and guides [14] suggests a velocity spectrum $n(v) \propto$ $v^{3} d v$ below cutoff, rather then a Maxwellian distribution $v^{2} d v$ because of absorption in the thick solid deuterium source. This number was lower than typical because these measurements were made with nonideal source conditions, low volume and high paradeuterium contamination. The cross sections obtained in this experiment, which are averaged over the UCN velocity spectrum, corresponds to the average velocity $v_{\text {av }} \simeq 4 \mathrm{~m} / \mathrm{s}$ (the UCN energy $\simeq 80 \mathrm{neV}$ ). Upscattering and capture have been measured simultaneously. The beam intensity during vanadium and hydrogen runs was monitored by our standard UCN monitor [14]. The $\gamma$ rays from neutron radiative capture in hydrogen and vanadium were measured in a $100 \%$ efficient (relative to a $7.6 \mathrm{~cm}$ diameter by $7.6 \mathrm{~cm}$ thick right circular cylinder of $\mathrm{NaI}$ ) high purity germanium detector (HPGe). The neutron detector for the upscattering measurement was a $30 \mathrm{~cm}$ long drift tubes [15] with 1.8 bar of ${ }^{3} \mathrm{He}$ and 1 bar of $\mathrm{CF}_{4}$. Both detectors were placed outside of the $7.5 \mathrm{~cm}$ diameter UCN guide upstream of the target location. The thicknesses of the targets, $0.25 \mathrm{~mm}$ of vanadium, and $1.6 \mathrm{~mm}$ of polyethylene, were chosen to absorb all neutrons that enter the materials. The target foils were backed by a nickel foil $\left[U_{F}(\mathrm{Ni}) \simeq 250 \mathrm{neV}\right]$ between them and the end of a UCN guide, reflecting any transmitted UCN neutrons back into the sample. The weak reflections from the vanadium and polyethylene surfaces are nearly equal and negligible for UCN with perpendicular energies higher than $\simeq 5 \mathrm{neV}$.

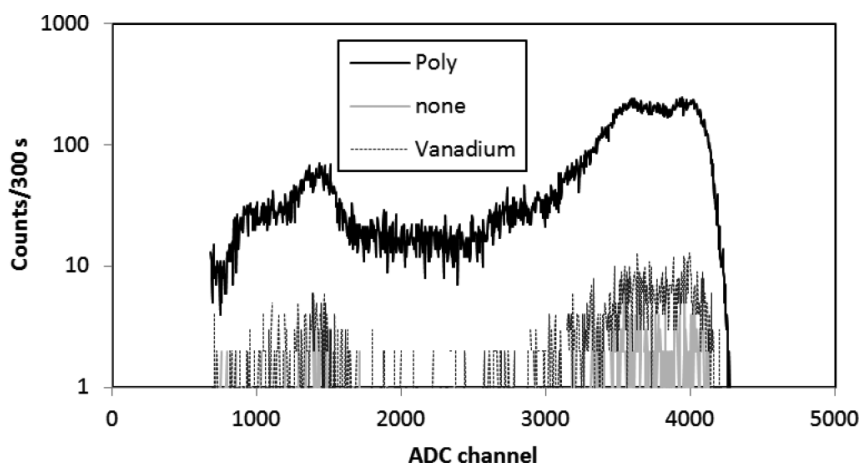

FIG. 1. Pulse height spectra from the $3 \mathrm{He}$ detector for the polyethylene, vanadium, and no target. The shoulder on the large pulse height peak and the low pulse height peak are due to wall effects in the detector.

In Fig. 1 pulse height spectra from the neutron detector for the two targets are compared to a run with no target. The upscatter rate with the polyethylene target is much larger than with the vanadium target. Both targets produce upscatter neutron rates significantly above the background, which was mostly due to cosmogenic thermal neutrons. The background associated with the proton beam was eliminated using time gates on the analog to digital converters, to reject events during the beam pulses. The relative neutron rates were calculated by integrating the spectra shown in Fig. 2 and subtracting the background measured with an empty target. The efficiencies of the ${ }^{3} \mathrm{He}$ detector for neutron spectra up scattered by $\mathrm{CH}_{2}$ and $\mathrm{V}$ have been modeled using spectral data of [16] and found to be equal with the accuracy of $2 \%$.

In the measurement with the HPGe detector a large number of $\gamma$-ray lines were observed with both the vanadium and the polyethylene foils. With the polyethylene foil many of the lines were due to up scattered neutrons capturing in the materials of the detector. With the vanadium foil there are a large number of capture lines extending up to $8 \mathrm{MeV}$. Two $\gamma$ ray lines have been used in the current analysis, the $2.225 \mathrm{MeV}$ $n$ - $p$ capture line and the $1.434 \mathrm{MeV} \gamma$ ray emitted in the decay of ${ }^{52} \mathrm{~V}$. The HPGe photopeak efficiencies for them in our geometry have been modeled by the MCNP5 code with the result $\epsilon(1.434)=1.37 \epsilon(2.225)$. The $\gamma$ rate from the decay of the isotope ${ }^{52} \mathrm{~V}$ produced by neutron capture builds up to equilibrium with a time constant given by the lifetime of ${ }^{52} \mathrm{~V}$ of 5.4 minutes. Equilibrium was ensured by waiting for two

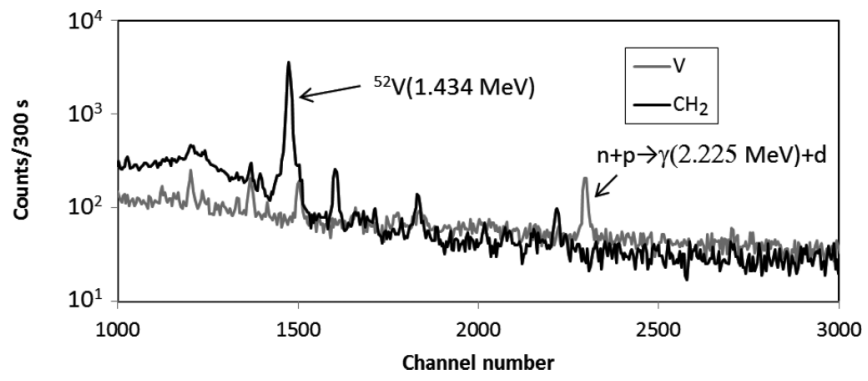

FIG. 2. $\gamma$-ray spectra obtained with UCN. The $\gamma$ line from $n-p$ capture with the polyethylene target and the $\gamma$ line from the product target ${ }^{52} \mathrm{~V}$ are labeled. 
TABLE I. Neutron $r_{n}$ and $\gamma$-ray $r_{a}$ count per $300 \mathrm{~s}$, their ratios $R(\mathrm{~V} / \mathrm{H})$ and the obtained $\mathrm{UCN}$ scattering cross sections $\sigma_{n}$ for the vanadium target and hydrogen in the polyethylene target. The $\gamma$-ray ratio has been corrected for the relative efficiency for the vanadium (1.44 MeV) and hydrogen capture $(2.22 \mathrm{MeV})$ lines. The absorption cross sections $\sigma_{a}$, used in the analysis, are obtained by applying the $1 / v$ law to thermal data from [10].

\begin{tabular}{lccc}
\hline \hline Targets and $R(\mathrm{~V} / \mathrm{H})$ & $r_{n}$ & $r_{a}$ & $\sigma_{n}(4 \mathrm{~m} / s), \mathrm{b}$ \\
\hline Vanadium $(\mathrm{V})$ & $19 \pm 5$ & $10590 \pm 11$ & $25 \pm 9$ \\
Polyethylene $(\mathrm{H})$ & $1869 \pm 22$ & $871 \pm 40$ & $2062 \pm 135$ \\
$R_{n}(\mathrm{~V} / \mathrm{H})$ & $0.010 \pm 0.003$ & & \\
$R_{a}(\mathrm{~V} / \mathrm{H})$ & & $12.16 \pm 0.7$ & \\
\hline \hline
\end{tabular}

lifetimes with the beam on target before starting measurements with the vanadium target. The resulting spectra are shown in Fig. 2. Rates were extracted by subtracting the background measured using the alternate target. The empty runs were not useful for these because of the large increase in background due to Compton events from other capture induced processes in the detector.

The data were analyzed as follows. Because all the incident $\mathrm{UCN}$ on $\mathrm{V}$ and $\mathrm{CH}_{2}$ are either captured or scattered, the relative contributions of these processes are given by the ratios $\sigma_{a} / \sigma_{t}$ and $\sigma_{n} / \sigma_{t}$, where the total cross section $\sigma_{t}$ is the sum $\sigma_{t}=\sigma_{n}+$ $\sigma_{a}$. The counting rates of the capture and neutron detectors, $r_{a}$ and $r_{n}$, corrected for efficiencies in the $\mathrm{V}$ and $\mathrm{H}$ targets are

$$
r_{a}=\frac{\sigma_{a}}{\sigma_{t}} \Phi S \epsilon_{a}, \quad r_{n}=\frac{\sigma_{n}}{\sigma_{t}} \Phi S \epsilon_{n},
$$

where $\Phi$ is the neutron fluence (the same for both targets after normalizing on the UCN monitor), $S$ is the target area (the same for both targets), $\epsilon_{a}$ and $\epsilon_{n}$ are the corresponding detectors efficiencies (including the solid angles) and we omitted, for simplicity, the indices of targets.

Introducing the ratios between vanadium and hydrogen count rates

$$
R_{a} \equiv \frac{r_{a}(\mathrm{~V})}{r_{a}(\mathrm{H})}=\frac{\sigma_{a}(\mathrm{~V})}{\sigma_{t}(\mathrm{~V})} \frac{\sigma_{t}(\mathrm{H})}{\sigma_{a}(\mathrm{H})},
$$

and

$$
R_{n} \equiv \frac{r_{n}(\mathrm{~V})}{r_{n}(\mathrm{H})}=\frac{\sigma_{n}(\mathrm{~V})}{\sigma_{t}(\mathrm{~V})} \frac{\sigma_{t}(\mathrm{H})}{\sigma_{n}(\mathrm{H})},
$$

we obtain the relationship between the measured $\mathrm{V}$ and $\mathrm{H}$ cross sections.

$$
\frac{\sigma_{a}(\mathrm{~V})}{\sigma_{n}(\mathrm{~V})}=\frac{R_{a}}{R_{n}} \frac{\sigma_{a}(\mathrm{H})}{\sigma_{n}(\mathrm{H})}
$$

This relationship has been pointed out previously by Bondarenko et al. [17] and used in a study of UCN interactions with hydrogen on metal surfaces. The relationships between $\sigma_{a}$ and $\sigma_{n}$ for each target separately are

$$
\frac{\sigma_{a}(\mathrm{H})}{\sigma_{n}(\mathrm{H})}=\frac{\left(1-R_{n}\right)}{\left(R_{a}-1\right)},
$$

for hydrogen and, after substitution it into Eq. (6),

$$
\frac{\sigma_{a}(\mathrm{~V})}{\sigma_{n}(\mathrm{~V})}=\frac{R_{a}}{R_{n}} \frac{\left(1-R_{n}\right)}{\left(R_{a}-1\right)}
$$

for vanadium.

The experimental results obtained from this analysis are given in Table I. From these data the UCN upscattering cross section on hydrogen, taken as the difference between the measured total scattering cross section $\sigma_{n}$ and the known (90 b) elastic $\sigma_{e l}$ cross section, is $\sigma_{\text {up }}(4 \mathrm{~m} / \mathrm{s})=1972 \pm 130 \mathrm{~b}$. Our measurements agrees with the recent TOF measurements of Pokotilovski et al. [13], but is lower than the range of values of 2200-2740 b obtained for a velocity of $4 \mathrm{~m} / \mathrm{s}$ from the ratio of upscattering to capture cross sections given by Korobkina et al. [18]. It should be noted that the MCNP calculation [8] of $\sigma_{\text {up }}$ for $\mathrm{CH}_{2}$ disagrees with our experimental result. Our result for vanadium is the first experimental confirmation of the extremely low UCN upscattering in V and, in this case, agrees with the theory of UCN heating by phonons in solids.

This work was performed under the auspices of the US Department of Energy under Contract No. DE-AC5206NA25396. D.J.S. is supported by the DOE Office of Science Graduate Fellowship Program (DOE SCGF), made possible in part by the American Recovery and Reinvestment Act of 2009, administered by ORISE-ORAU under contract no. DE-AC05-06OR23100.
[1] S. K. Lamoreaux and R. Golub, J. Phys. G: Nucl. Part. Phys. 36, 104002 (2009).

[2] Fred E. Wietfeldt and Geoffrey L. Greene, Rev. Mod. Phys. 83, 1173 (2011).

[3] Hartmut Abele, Prog. Part. Nucl. Phys. 60, 1 (2008).

[4] Valerii V. Nesvizhevsky, Phys. Usp. 53, 645 (2010).

[5] D. Dubbers and M. G. Schmidt, Rev. Mod. Phys. 83, 111 (2011).

[6] I. I. Gurevich and L. V. Tarasov, Low-Energy Neutron Physics (North-Holland Publishing Company, Amsterdam, 1968).
[7] X-5-Monte-Carlo-team, MCNP - a General Monte Carlo N-Particle Transport Code, Version 5, Report No. LA-UR-03198, Los Alamos National Laboratory, Los Alamos 2003.

[8] D. E. Cullen, L. F. Hansen, E. M. Lent, and E. F. Plecharty, Report UCRL-ID-153656, Lawrence Livermore National Laboratory, Livermore, available from http://www. llnl.gov/tid/Library.html.

[9] J. A. Polo and J. M. Robson, Phys. Rev. C 27, 133 (1983).

[10] S. F. Mughabghab, Atlas of Neutron Resonances: Resonance Parameters and Thermal Cross Sections $Z=1-100$ (Elsevier, Amsterdam, 2006). 
[11] W. Dilg and W. Mannhart, Z. Phys. 266, 157 (1974).

[12] S. W. Zhukov, V. L. Kuznetsov, V. I. Morozov, Yu. N. Panin, A. I. Fomin, and S. M. Chernyavskii, JETP Lett. 57, 464 (1993).

[13] Yu. N. Pokotilovski, M. I. Novopoltsev, P. Geltenbort, and T. Brenner, Instrum. Exp. Tech. 54, 16 (2011).

[14] A. Saunders, M. Makela, Y. Bagdasarova et al., Rev. Sci. Instrum. 84, 013304 (2013).
[15] Zhehui Wang, C. L. Morris, M. Makela et al., Nucl. Instrum. Methods Phys. Res. A 605, 430 (2009).

[16] E. I. Sharapov, C. L. Morris, and M. Makela et al., Phys. Rev. B (to be published).

[17] L. Bondarenko, S. Chernyavsky, A. Fomin, P. Geltenbort, V. Morozov, and S. Shilkin, Physica B 234, 1189 (1997).

[18] E. Korobkina and R. Golub, J. Butterworth, P. Geltenbort, and S. Arzumanov, Phys. Rev. B 70, 035409 (2004). 